\title{
BMJ Open Comparing efficacy and safety in catheter ablation strategies for atrial fibrillation: protocol of a network meta- analysis of randomised controlled trials
}

Emmanouil Charitakis (10 , ${ }^{1}$ Lars O Karlsson, ${ }^{1}$ Kostantinos Rizas, ${ }^{2}$ Henrik Almroth, ${ }^{1}$ Anders Hassel Jönsson, ${ }^{1}$ Jonas Schweiler, ${ }^{3}$ Skevos Sideris, ${ }^{4}$ Dimitrios Tsartsalis, ${ }^{5}$ Elena Dragioti (iD , ${ }^{6}$ Anna Chaimani ${ }^{7}$

To cite: Charitakis $\mathrm{E}$, Karlsson L0, Rizas K, et al. Comparing efficacy and safety in catheter ablation strategies for atrial fibrillation: protocol of a network metaanalysis of randomised controlled trials. BMJ Open 2020;10:e041819. doi:10.1136/ bmjopen-2020-041819

- Prepublication history and supplemental material for this paper are available online. To view these files, please visit the journal online (http://dx.doi org/10.1136/bmjopen-2020041819).

Received 17 June 2020 Revised 15 September 2020 Accepted 21 October 2020

Check for updates

(C) Author(s) (or their employer(s)) 2020. Re-use permitted under CC BY-NC. No commercial re-use. See rights and permissions. Published by BMJ.

For numbered affiliations see end of article.

Correspondence to Dr Emmanouil Charitakis; emmanouil.charitakis@liu.se

\section{ABSTRACT}

Introduction Atrial fibrillation (AF) is the most common sustained arrhythmia. Catheter ablation (CA) of AF is an increasingly offered therapeutic approach, primary to relieve AF-related symptoms. Despite the development of new ablation approaches, there is no consensus regarding the most efficient ablation strategy. The objective of this network meta-analysis (NMA) is to compare the efficacy and safety of all different CA approaches for the treatment of patients with paroxysmal (PAF) and non-PAF (non-PAF). Methods and analysis We will perform a systematic search to identify randomised controlled trials of different CA approaches for the treatment of PAF and non-PAF, through the final search date of 1 March 2020. Information sources will include major bibliographic databases (MEDLINE, Web of Science and CENTRAL) and clinical trial registries. Our primary outcomes will be the efficacy (recurrence-free survival) and safety of different CA approaches for the treatment of AF. Secondary outcomes will be all-cause mortality and procedural time. An NMA will be performed to determine the relative effects of different catheter ablation approaches (such as pulmonary vein isolation alone or in combination with ablation lines, ablation of complex fractionated atrial electrograms, etc). In PAF, a separate analysis will be performed including different energy sources (such as radiofrequency, cryogenic and laser energy). Risk of bias assessment and sensitivity analyses will be conducted to assess the robustness of the findings to potential bias.

Ethics and dissemination № ethical approval will be needed because data are collected from previous studies. The results will be presented through peer-review journals and conference presentation.

PROSPERO registration number CRD42020169494.

\section{INTRODUCTION}

Atrial fibrillation (AF) is the most common cardiac arrhythmia, affecting $3 \%$ of the adult population. ${ }^{1}$ It is estimated that $25 \%$ of adults will develop AF in Europe and USA. ${ }^{2}$ Despite therapeutic advancements, AF remains one of the major causes of stroke, heart failure and sudden death ${ }^{2}$ and constitutes a significant
Strengths and limitations of this study

- Network meta-analysis (NMA) is a strong and robust statistical method that allows for direct and indirect comparisons (through one or more intermediate comparators) and their combination.

- NMA concerning the efficacy and safety of different catheter ablation approaches for patients with atrial fibrillation (AF).

- Given the high prevalence of $A F$ and the limited therapeutic options, we expect that the results of this NMA will enhance evidence to guide both treatment decisions and future guidelines concerning catheter ablation approach for patients with AF.

- This NMA is restricted to randomised controlled trials (RCTs).

- Taking into consideration the nature of the RCTs included in this NMA, blinding of the care provider (operator) is not feasible.

burden for the health system, calculated to account for approximately $1 \%$ of total healthcare spending in $\mathrm{UK}^{3}$

Catheter ablation has become an important treatment option for symptomatic patients with paroxysmal and non-paroxysmal $\mathrm{AF}$ $(\mathrm{PAF} /$ non-PAF $) .^{2}$ A recent meta-analysis showed that CA is associated with lower allcause mortality and reduced recurrences of atrial arrhythmia compared with medical treatment. $^{4}$

Since the initial description of the initiation of AF by ectopic beats originating from the pulmonary veins by Haïssaguerre et $a l^{5}$ pulmonary vein isolation (PVI) has been the cornerstone of any $\mathrm{AF}$ ablation procedure irrespective of the type of $\mathrm{AF}$ and patient characteristics. ${ }^{6}$

During the last 18 years, various ablation strategies have been introduced and implemented into clinical practice as stand-alone 
strategies or as a complement to PVI, however, the scientific evidence for these strategies have been equivocal. The most often implemented strategies included linear lesions, substrate modification, electrocardiogram (EGM)-based approaches (among others, ablation of complex fractionated atrial electrograms), as well as identification and ablation of trigger sites and ganglia-plexi (GP). ${ }^{6}$

Furthermore, different energy sources have been used for the isolation of pulmonary veins. The most frequent sources used are radiofrequency energy, mostly applied in a point-by-point manner or by a 'single shot device', and the cryogenic energy applied with a balloon in a single-step model. ${ }^{7}$ In recent years, laser balloons have been introduced as an alternative method to radiofrequency and cryogenic energy for PVI isolation. Different approaches have been called into question by different randomised control trials failing to demonstrate beneficial outcome.

Network meta-analysis (NMA) is a statistical method that allows for direct and indirect comparisons (through one or more intermediate comparators) and their combination. ${ }^{8}$ NMA can incorporate randomised controlled trials (RCTs) of several different treatments/interventions with a given endpoint and has already been applied widely in several medical fields. ${ }^{9}$

The objective of this systematic review and NMA is to compare the efficacy and safety of all different CA approaches (such as PVI, PVI and substrate modification, PVI and Ganglion plexi ablation, PVI and EGM-based approach, EGM-based approach alone, etc) for the treatment of patients with PAF and non-PAF.

A separate analysis for patients with PAF will additionally be performed. This analysis will consider comparisons between different energy sources (radiofrequency (point-by-point or dual-cycled phase), cryoballoon or laser balloon technologies, etc) for the isolation of the pulmonary veins.

\section{METHODS}

\section{Study design and registration}

This protocol has been prepared according to the Preferred Reporting Items for Systematic Reviews and Meta-Analyses Protocols (PRISMA) recommendations (online supplemental file 1$)^{10}$ and Cochrane recommendations. ${ }^{11}$ The PRISMA extension statement for NMA will be used to prepare the final report of this study. ${ }^{12}$

Eligibility criteria were developed using the PICOS framework and are reported in the following sections and summarised in table 1.

\section{Types of studies and participants}

This review will only include RCTs with a parallel group study design comparing different CA approaches or a CA approach versus antiarrhythmic drugs or rate control therapy. RCTs of different designs such as crossover trials, quasi-RCTs, factorial and cluster RCTs or quasi-experimental studies (controlled before and after studies) will be excluded.

Studies with patients over 18 years of age of both sexes with PAF or non-PAF according to the current guidelines will be included. ${ }^{2}$

We will exclude studies if they (1) used a study design other than RCT, (2) included patients with prior ablation, surgical ablation and rate control via atrioventricular node ablation.

There will be no language restrictions of the literature search, however, RCTs that are not possible to translate into English will be excluded from quantitative analysis.

\section{Type of interventions}

We are interested in comparing all previously published CA approaches evaluated in RCTs. We will include interventions such as PVI as standalone, non-PVI approaches (such as EGM-based approach, GP approach, only ablation linear lesion approach), different ablation approaches complementary to PVI (such as PVI and EGM-based approach, PVI and linear lesions, PVI and substrate modification and PVI and GP, etc) and medical therapy (table 2).

A separate analysis for patients with PAF is to be performed, including different energy sources used for PVI (wide antral circumferential ablation, cryoballoon ablation, laser balloon ablation, etc), as well as previously mentioned approaches (table 2).

We assume that for each of the planned networks of interventions depicted in figures 1 and 2, any patient who meets all inclusion criteria is equally likely to be randomised in any of these interventions.

Table 1 Summary of PICOS eligibility criteria

\begin{tabular}{lll}
\hline & Inclusion criteria & Exclusion criteria \\
\hline Population & $\begin{array}{l}\text { Adults ( } \geq 18 \text { years) with paroxysmal or non-paroxysmal atrial } \\
\text { fibrillation }\end{array}$ & $\begin{array}{l}\text { Patients with prior ablation, surgical ablation and rate } \\
\text { control via atrioventricular node ablation. }\end{array}$ \\
\hline Intervention & $\begin{array}{l}\text { Cathether ablation } \\
\text { Comparison }\end{array}$ & $\begin{array}{l}\text { A different eligible catheter ablation approaches compared with } \\
\text { a control (another catheter ablation treatment or antiarrhythmic } \\
\text { drugs) }\end{array}$ \\
Outcome & $\begin{array}{l}\text { Efficacy (arrhythmia-free survival) and safety (peri procedural and } \\
\text { postprocedural complications) }\end{array}$ & \\
\hline Study type & Randomised clinical trials & Non-randomised trials \\
\hline
\end{tabular}


Table 2 Examples of catheter ablation strategies comparisons in the whole population analysis and in the subgroup of patients with paroxysmal atrial fibrillation

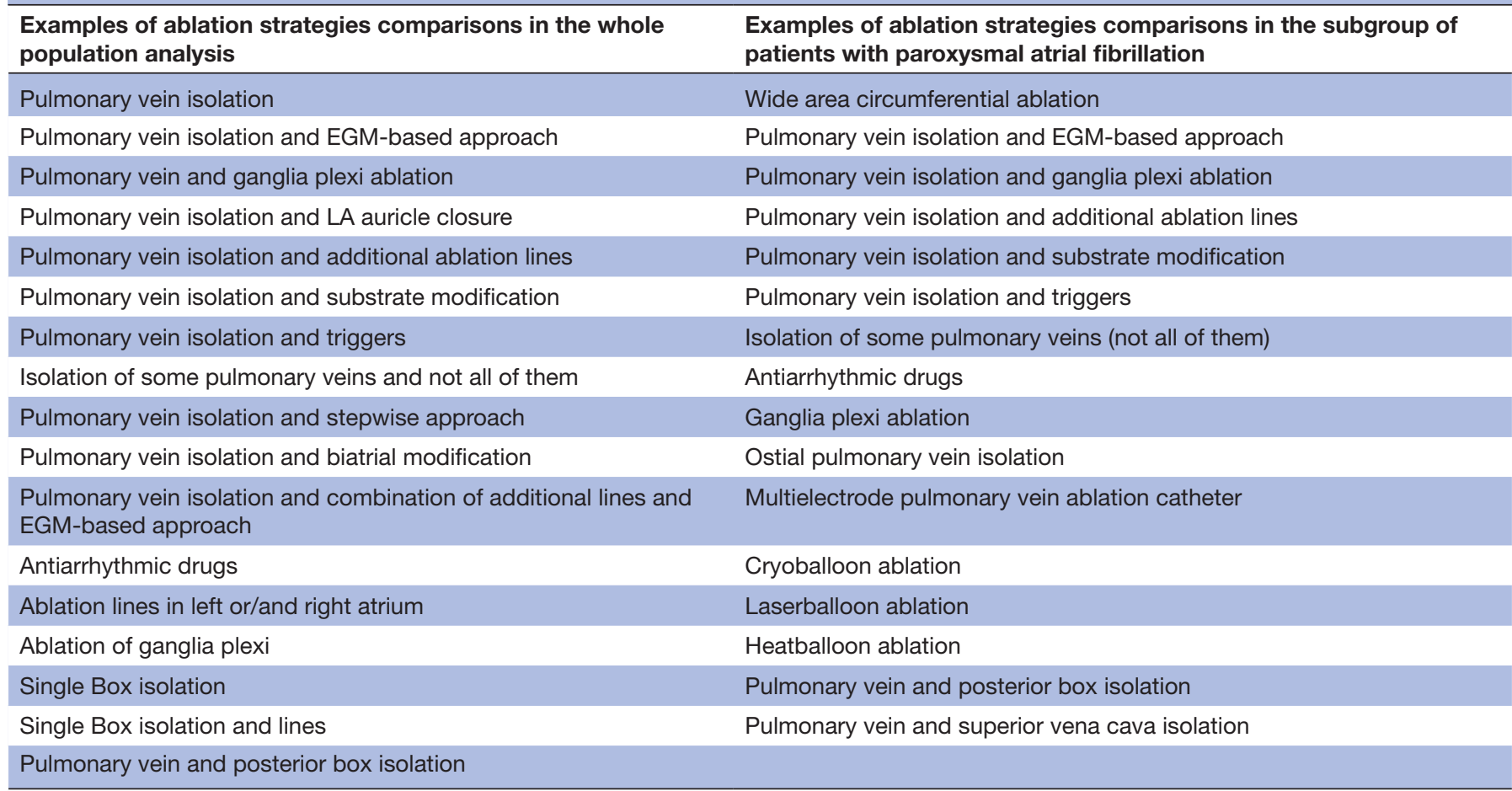

EGM, electrocardiogram; LA, left atrial.

\section{Outcomes and measures}

RCT of CA approaches for the treatment of AF are usually small and the data distribution is difficult to assess. In this review, priority will be given to the analysis of two dichotomous variables, efficacy and safety.
In order to assess efficacy due to relative low mortality rate 1 year after CA, most RCTs performed use neither hard endpoints (mortality, hospitalisation, other cardiovascular events) nor arrhythmia burden as their primary endpoints. Therefore, we decided to

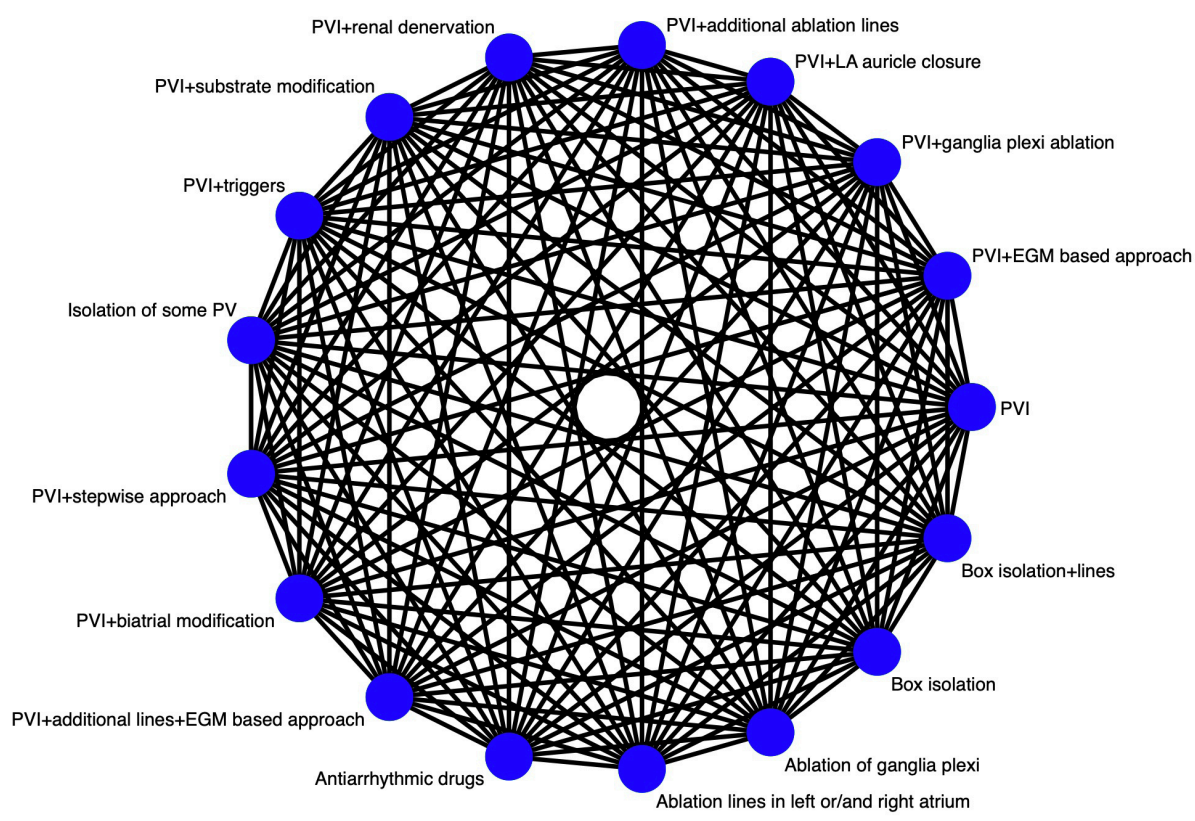

Figure 1 Provisional network graph for paroxysmal and non-paroxysmal atrial fibrillation analysis. EGM, electrocardiogram; $\mathrm{LA}$, left atrial; PVI, pulmonary vein isolation. 


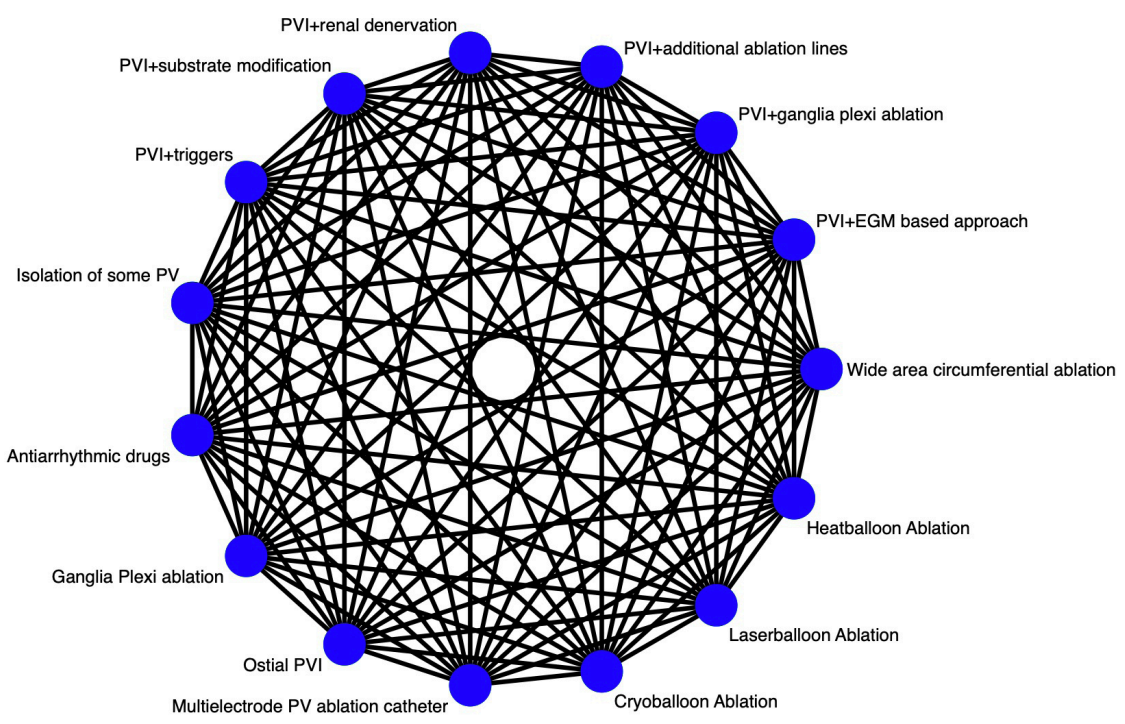

Figure 2 Provisional network graph for paroxysmal atrial fibrillation analysis. EGM, electrocardiogram; PVI, pulmonary vein isolation.

use recurrence rate as the primary outcome for this review.

\section{Primary outcomes}

- Efficacy: Recurrence after CA for AF during a follow-up of no less than 6 months.

Recurrence after CA for AF is defined as any episode of $\mathrm{AF}$ or atrial arrhythmia with a duration $>30 \mathrm{~s}$ recorded on ECG, ambulatory ECG, device (pacemaker, defibrillator) or implantable loop recording (ILR). The $30 \mathrm{~s}$ limit was determined by consensus to be a reasonable threshold by which most would consider calling AF an atrial arrhythmia episode. $^{13}$

- Safety: All reported periprocedural complications and complications related to the procedure during the follow-up after CA.

Serious as well as minor reported complications related to the procedure are to be included irrespective of whether they were periprocedural or occurred during the follow-up.

Secondary outcomes

- All-cause mortality.

All the reported cases of death from the time of randomisation to the end of each study follow-up.

- Procedural time (continuous variable).

The procedural time is defined as the time from catheterisation to catheter retrieval, and expressed as mean \pm SD.

\section{Search strategy and study selection}

A comprehensive search on RCTs will be conducted by EC and ED independently using PubMed, the Cochrane
Central Register of Controlled Trials (CENTRAL), and the Web of Science, comparing different CA approaches or CA to medical therapy, through the final search date of 1 March 2020. We will use the following keywords in various combinations: atrial fibrillation, auricular fibrillation, AF, radiofrequency ablation, catheter ablation, ablation, CA, cryoablation, cryoballoon, PVI with no date restrictions. Details of search terms and strategy will be provided in the supporting information (online supplemental file 2).

We will also hand search the reference lists of included studies and previously published systematic reviews for additional studies which would not have been identified in the original search.

Two persons (EC and ED) will independently review abstracts retrieved by the search. In case both reviewers agree that an RCT does not meet eligibility criteria, it will be excluded. We will then obtain the full text of all remaining articles and check the eligibility criteria in order to determine which, if any, to exclude at this stage. Disagreements will be resolved by discussion with a third member of the review team.

\section{Data extraction}

Two authors (EC and ED) will independently read each article and evaluate the completeness of the data abstraction, confirm the risk of bias (RoB) and the indirectness. Data abstraction will be performed independently by two investigators (EC and ED) in a structured manner to ensure data consistency and appraisal for each study. Any disagreements will be resolved by consensus or consultation with a third reviewer (AC) if disagreements persist. 
We will abstract data based on the study characteristics (including the first author's name, publication year, enrolment period, trial design), patient characteristics (such as age, sex, type of AF, background factors, etc), intervention details (such as different ablation approaches, fluoroscopy time, time for follow-up, detection of AF, blanking period, etc) and outcome measures. Whenever a full text is unavailable the original authors will be contacted for data request

\section{RoB assessment}

We will use the Cochrane Collaboration RoB tool for randomised trials (RoB V.2) ${ }^{14}$ to rate the quality of the included RCTs. RoB V.2 is structured into a fixed set of domains of bias, focusing on different aspects of trial design, conduct and reporting. Within each domain, a series of questions ('signalling questions') aim to elicit information about features of the trial that are relevant to RoB. A proposed decision about the RoB arising from the intended intervention, missing outcome data, measurement and selective reporting is generated by an algorithm, based on answers to the signalling questions. A decision can be of 'low' or 'high' RoB or can express 'some concerns'. 14

It is important to note that the included RCTs in this NMA concern different types of interventions and blinding of the care providers (operators) is not feasible. However, blinding of participants is still feasible and will be considered on rating the RoB of each study.

\section{Dealing with missing data}

In order to deal with missing data, we plan to contact the authors of the original studies. However, after contacting the authors at least two times, studies with insufficient data will be excluded. Missing outcome data will be assessed as previously mentioned with the use of ROB V.2 tool and graded as having low, high or medium RoB For dichotomous outcomes: if a study reported 0 events in one treatment arm, we would add a 0.5 to the numerator and a 1 to the denominator. If studies report 0 events in all treatments arms, they will be excluded. ${ }^{15}{ }^{16}$ For continuous outcomes: missing SDs will be calculated from SEs, CIs or other measures. ${ }^{1718}$ Mean and SD will be calculated from median and IQR using specific formula. ${ }^{1920}$

\section{Data synthesis}

\section{Pairwise meta-analysis}

We will generate descriptive statistics for trial and study population characteristics (clinical or methodological) across all eligible trials, and across the available direct comparisons to investigate potential discrepancies across trial and population characteristics.

For each comparison with at least two studies, we will first perform random effects pairwise meta-analysis. ${ }^{21}$ The risk ratio will be calculated as the effect size for dichotomous outcomes and the mean difference for continuous outcomes, both with a $95 \%$ CI. We will investigate the potential for statistical heterogeneity using visual inspection of the forest plots, as well as, considering the magnitude of the between-study variance $\left(\tau^{2}\right)$ and the $\mathrm{I}^{2}$ statistic. $^{22}$

\section{Evaluation of transitivity}

Transitivity is the fundamental assumption of NMA and implies that one can validly learn about $\mathrm{B}$ versus $\mathrm{C}$ through A using studies comparing A versus B and studies comparing A versus C. We will carefully evaluate the characteristics of the interventions when they are included in studies involving different sets of interventions. We will also investigate the distribution of the potential effect modifiers, such as age, sex, the presence of hypertension, diabetes mellitus, structural heart disease, ischaemic heart disease, as well as, the left atrial dimensions and the left ventricle ejection fraction, across the available direct comparisons and assess how similar they are.

\section{Network meta-analysis}

If there is no evidence for important intransitivity, we will then perform a random effects network meta-analysis to synthesise information on all available interventions for each outcome simultaneously. We will perform the analyses and produce tabular and graphical depictions in Stata V.16 using the network and network graphs packages and in $\mathrm{R}$ using the netmeta package. ${ }^{23-26} \mathrm{~A}$ common heterogeneity parameter across all comparisons will be assumed for each network of interventions. We will rank the competing interventions using the surface under the cumulative ranking curve (SUCRA) ${ }^{27}$ for the primary outcomes. A SUCRA of $100 \%$ implies that a treatment is certain to be the best, and an SUCRA of $0 \%$ that it is certain to be the worst for a particular outcome.

Since most interventions listed above are combinations of other interventions, we also intend to run a componentlevel analysis given that sufficient number of studies per component will be available. ${ }^{28} 29$

\section{Assessment of statistical incoherence}

We will evaluate the assumption of statistical coherence (ie, that direct and indirect evidence are in agreement) using two approaches: the side-splitting method and the design-by-treatment interaction model. The former evaluates incoherence for every comparison with available direct evidence and the latter tests incoherence in the entire network jointly.

\section{Subgroup analyses}

- Depending on AF detection device

We will perform a separate analysis comprising RCTs that used a more sensitive method for the detection of $\mathrm{AF}$ that is, ILR, ambulatory ECGs with recording times $\geq 7$ days.

- Depending on Antiarrhythmic drugs (AAD) or reablation allowance during the follow-up.

Some RCTs allow the use of AAD during follow-up. This condition can have an impact on the rate of recurrence as the AAD reduce the risk of recurrent AF. Hence, a separate analysis will be performed comprising studies that do 
not allow AAD during follow-up in order to have a better homogeneity in the population.

- Depending on follow-up duration.

A separate analysis will be performed comprising studies with longer follow-up period $\geq 12$ months.

- In patients with non-PAF.

Non-PAF comprises persistent $\mathrm{AF}$ and long-persistent $\mathrm{AF}$ that is, $\mathrm{AF}$ that lasts longer than 7 days. $^{2}$

As mentioned above (type of intervention section) a separate analysis will be performed concerning patients with PAF, where different sources for isolation of the PV will be included in addition to previously mentioned approaches for $\mathrm{CA}$ of $\mathrm{AF}$.

\section{Meta-regression analysis}

Meta-regression analyses will be performed for clinically relevant subgroups or in the presence of important heterogeneity or incoherence. ${ }^{18}$ With this analysis, we will be able to examine the impact of important effect modifiers on our results such as age, gender, hypertension, diabetes, the existence of congestive heart failure and structural disease, as well as the dimensions of the LA, the duration of the follow-up.

\section{Across-study biases}

The evaluation of small-study effects will be performed using the comparison-adjusted funnel plot. ${ }^{25}$ In case funnel plot asymmetry is present, we will perform network meta-regression models testing the significance of the asymmetry. ${ }^{30}$ To assess the potential of publication bias we will primarily consider how likely it would be that published study results have not been captured by our search strategy. If the risk for publication bias will be considerable, we will apply selection models that model the probability of study being published. ${ }^{31}$

\section{Sensitivity analyses}

The Sensitivity of our conclusions will be tested for the primary outcomes will be tested by analysing ${ }^{1}$ only studies with low RoB (as reported in RoB section) ${ }^{2}$ only studies with head to head comparison of two different CA ablation approaches excluding studies that have drugs as comparing $\mathrm{arm}^{3}$ Only studies that include patients with non-PAF.

\section{Quality of evidence}

The overall quality of the evidence for the main outcomes will also be assessed using the Confidence in Network Meta-Analysis (CINeMA) tool (https://cinema.ispm. unibe.ch). CINeMA allows for a quality of evidence judgement of every comparison in the network. For study limitations and indirectness the judgements take into account the relative contributions of direct and indirect studies in the estimation. ${ }^{32}$ For imprecision, heterogeneity and incoherence, the tool uses the concept of the minimally clinically important effect size and constructs the 'range of equivalence' between two intervention. Then, judgements about the three domains consider whether uncertainty intervals lie within this range.

\section{Ethics and dissemination}

This review does not require ethical approval or consent to participate. The findings from this systematic review will be published in a peer-reviewed scientific journal, and data set will be made freely available on request.

\section{Patient and public involvement}

This study is an NMA based on previous published data, concerning different catheter ablation approaches and/ or choice of different energy sources. While we value the involvement of patients and public throughout the research study cycle, for this study, it was not deemed essential to involve them in this cycle, as this study is further exploring existing data.

\section{DISCUSSION}

$\mathrm{AF}$ is the most common sustained arrhythmia with a global prevalence of 33.5 million persons. ${ }^{33} \mathrm{CA}$ is an increasingly offered therapeutic approach, primarily performed in order to relieve AF-related symptoms. ${ }^{34}$ Even though new ablation approaches emerge, at present there is no consensus regarding the most efficient ablation strategy. ${ }^{35}$

To the best of our knowledge, this is the first NMA planned in order to compare the efficiency and safety of different CA strategies in patients with AF. The results from this NMA will provide an important evidence base for clinicians to guide treatment decisions by providing a comparative assessment of a wide range of different CA approaches. This will help in the development of a better CA strategy in patients with different types of AF. Numerous factors must be considered prior to CA of AF, such as individual patient suitability and patient preferences, and reliable information on the efficiency of different CA approaches as well as an assessment of how bias-free these results might be are fundamental points in guiding these decisions.

Furthermore, given the high prevalence of AF and the limited therapeutic options, we expect the results of the NMA to be of considerable interest to clinicians, academics and authors of guidelines. Hence, we plan to disseminate the findings widely through academic publications, conference presentations and communication with healthcare providers.

\section{Author affiliations}

'Department of Cardiology and Department of Health, Medicine and Caring Sciences, Linköping University Hospital, Linköping, Sweden

${ }^{2}$ Department of Cardiology, LMU München, Munchen, Germany

${ }^{3}$ Heart and Vascular Theme, Karolinska University Hospital, Stockholm, Sweden ${ }^{4}$ Department of Cardiology, Hippokration Hospital, National and Kapodistrian University of Athens, Athens, Attica, Greece

${ }^{5}$ Department of Clinical Physiology, Linköping University Hospital, Linköping, Sweden

${ }^{6}$ Pain and Rehabilitation Center and Department of Health and Caring Sciences, Linköping University, Linköping, Sweden

${ }^{7}$ Research Center of Epidemiology and Statistics (CRESS-U1153), Univeristé de Paris, Paris, Île-de-France, France

Twitter Emmanouil Charitakis @mcharitakis and Dimitrios Tsartsalis @dtsartsal 
Contributors EC and ED were responsible for the conception and design of the study and for writing the initial protocol draft. ED and AC developed the statistical analysis plan. ED, AC, LOK, KR, HA, AHJ, JS, DT and SS provided critical revisions of all aspects of the review. The final protocol has been approved by all authors.

Funding The authors have not declared a specific grant for this research from any funding agency in the public, commercial or not-for-profit sectors.

Competing interests None declared.

Patient consent for publication Not required.

Provenance and peer review Not commissioned; externally peer reviewed.

Supplemental material This content has been supplied by the author(s). It has not been vetted by BMJ Publishing Group Limited (BMJ) and may not have been peer-reviewed. Any opinions or recommendations discussed are solely those of the author(s) and are not endorsed by BMJ. BMJ disclaims all liability and responsibility arising from any reliance placed on the content. Where the content includes any translated material, BMJ does not warrant the accuracy and reliability of the translations (including but not limited to local regulations, clinical guidelines, terminology, drug names and drug dosages), and is not responsible for any error and/or omissions arising from translation and adaptation or otherwise.

Open access This is an open access article distributed in accordance with the Creative Commons Attribution Non Commercial (CC BY-NC 4.0) license, which permits others to distribute, remix, adapt, build upon this work non-commercially, and license their derivative works on different terms, provided the original work is properly cited, appropriate credit is given, any changes made indicated, and the use is non-commercial. See: http://creativecommons.org/licenses/by-nc/4.0/.

\section{ORCID iDs}

Emmanouil Charitakis http://orcid.org/0000-0002-2514-5324

Elena Dragioti http://orcid.org/0000-0001-9019-4125

\section{REFERENCES}

1 Friberg L, Bergfeldt L. Atrial fibrillation prevalence revisited. $J$ Intern Med 2013;274:461-8.

2 Kirchhof P, Benussi S, Kotecha D, et al. 2016 ESC guidelines for the management of atrial fibrillation developed in collaboration with EACTS. Eur Heart J 2016;37:2893-962.

3 Stewart S, Murphy NF, Murphy N, Walker A, et al. Cost of an emerging epidemic: an economic analysis of atrial fibrillation in the UK. Heart 2004;90:286-92.

4 Asad ZUA, Yousif A, Khan MS, et al. Catheter ablation versus medical therapy for atrial fibrillation. Circulation 2019;12:e007414.

5 Haïssaguerre M, Jaïs P, Shah DC, et al. Spontaneous initiation of atrial fibrillation by ectopic beats originating in the pulmonary veins. N Engl J Med 1998;339:659-66.

6 Calkins H, Hindricks G, Cappato R, et al. 2017 HRS/EHRA/ECAS/ APHRS/SOLAECE expert consensus statement on catheter and surgical ablation of atrial fibrillation: Executive summary. Heart Rhythm 2017;14:e445-94.

7 Kuck K-H, Brugada J, Fürnkranz A, et al. Cryoballoon or radiofrequency ablation for paroxysmal atrial fibrillation. $N$ Engl $J$ Med 2016;374:2235-45.

8 Cipriani A, Higgins JPT, Geddes JR, et al. Conceptual and technical challenges in network meta-analysis. Ann Intern Med 2013:159:130-7.

9 Elliott WJ, Meyer PM. Incident diabetes in clinical trials of antihypertensive drugs: a network meta-analysis. Lancet 2007;369:201-7.

10 Moher Det al. Preferred reporting items for systematic reviews and meta-analyses: the PRISMA statement. Ann Intern Med 2009;151:264.

11 Chaimani A, Caldwell DM, Li T, et al. Additional considerations are required when preparing a protocol for a systematic review with multiple interventions. J Clin Epidemiol 2017;83:65-74.
12 Hutton B, Salanti G, Caldwell DM, et al. The PRISMA extension statement for reporting of systematic reviews incorporating network meta-analyses of health care interventions: checklist and explanations. Ann Intern Med 2015;162:777-84.

13 Calkins H, Kuck KH, Cappato R, et al. 2012 HRS/EHRA/ECAS expert consensus statement on catheter and surgical ablation of atrial fibrillation: recommendations for patient selection, procedural techniques, patient management and follow-up, definitions, endpoints, and research trial design. Europace 2012;14:528-606.

14 Sterne JAC, Savović J, Page MJ, et al. Rob 2: a revised tool for assessing risk of bias in randomised trials. BMJ 2019;366:14898.

15 Sweeting MJ, Sutton AJ, Lambert PC. What to add to nothing? use and avoidance of continuity corrections in meta-analysis of sparse data. Stat Med 2004;23:1351-75.

16 Bradburn MJ, Deeks JJ, Berlin JA, et al. Much ado about nothing: a comparison of the performance of meta-analytical methods with rare events. Stat Med 2007;26:53-77.

17 Furukawa TA, Salanti G, Atkinson LZ, et al. Comparative efficacy and acceptability of first-generation and second-generation antidepressants in the acute treatment of major depression: protocol for a network meta-analysis. BMJ Open 2016;6:e010919.

18 Higgins JPT TJ, Chandler J, Cumpston M, et al. Cochrane handbook for systematic reviews of interventions version 6.0 Cochrane, 2019. Available: www.training.cochrane.org/handbook

19 Wan X, Wang W, Liu J, et al. Estimating the sample mean and standard deviation from the sample size, median, range and/or interquartile range. BMC Med Res Methodol 2014;14:135.

20 Hozo SP, Djulbegovic B, Hozo I. Estimating the mean and variance from the median, range, and the size of a sample. BMC Med Res Methodol 2005;5:13.

21 DerSimonian R, Laird N. Meta-analysis in clinical trials. Control Clin Trials 1986;7:177-88.

22 Higgins JPT, Thompson SG, Deeks JJ, et al. Measuring inconsistency in meta-analyses. BMJ 2003;327:557-60.

23 White IR. Network meta-analysis. Stata J 2015;15:951-85.

24 Chaimani A, Salanti G. Visualizing assumptions and results in network meta-analysis: the network graphs package. Stata $J$ 2015;15:905-50.

25 Chaimani A, Higgins JPT, Mavridis D, et al. Graphical tools for network meta-analysis in STATA. PLoS One 2013;8:e76654.

26 Rücker G, Krahn U, König J, et al. netmeta: network meta-analysis using Frequentist methods. R package version 1.2-1, 2020. Available: https://CRAN.R-project.org/package=netmeta

27 Salanti G, Ades AE, loannidis JPA. Graphical methods and numerical summaries for presenting results from multipletreatment meta-analysis: an overview and tutorial. J Clin Epidemiol 2011;64:163-71.

28 Rücker G, Petropoulou M, Schwarzer G. Network meta-analysis of multicomponent interventions. Biom J 2020;62:808-21.

29 Welton NJ, Caldwell DM, Adamopoulos E, et al. Mixed treatment comparison meta-analysis of complex interventions: psychological interventions in coronary heart disease. Am J Epidemiol 2009;169:1158-65.

30 Chaimani A, Salanti G. Using network meta-analysis to evaluate the existence of small-study effects in a network of interventions. Res Synth Methods 2012;3:161-76.

31 Mavridis D, Welton NJ, Sutton A, et al. A selection model for accounting for publication bias in a full network meta-analysis. Stat Med 2014;33:5399-412.

32 Salanti G, Del Giovane C, Chaimani A, et al. Evaluating the quality of evidence from a network meta-analysis. PLoS One 2014;9:e99682.

33 Chugh SS, Havmoeller R, Narayanan K, et al. Worldwide epidemiology of atrial fibrillation: a global burden of disease 2010 study. Circulation 2014;129:837-47.

34 Arbelo E, Brugada J, Hindricks G, et al. The atrial fibrillation ablation pilot study: a European survey on methodology and results of catheter ablation for atrial fibrillation conducted by the European heart rhythm association. Eur Heart J 2014;35:1466-78.

35 Kirchhof $\mathrm{P}$, Calkins $\mathrm{H}$. Catheter ablation in patients with persistent atrial fibrillation. Eur Heart J 2017;38:20-6. 